\title{
MUSGOS DA ILHA DE MARAJÓ - II - MUNICÍPIO DE ANAJÁS PARÁ, BRASIL
}

\author{
Regina C. L. LISBOA', Maria Josiane L. de LIMA², Ubirajara N. MACIEL ${ }^{\prime}$
}

RESUMO - No município de Anajás, Ilha de Marajó, situado no estado do Pará, foram realizadas coletas de Bryophyta (musgos), em continuidade ao projeto que tem como objetivo conhecer a brioflora da ilha. Foram identificadas 34 espécies, distribuídas em 25 gêneros e 17 familias. Destas, Calymperaceae e Sematophyllaceae destacaram-se pela diversidade especifica, com seis e cinco espécies, respectivamente. Os musgos Syrrhopodon leprieurii Mont., Fissidens elegans Brid., Isopterygium subbrevisetum (Hampe) Broth. e Meiothecium boryanum (C. Müll.) Mitt., são apresentados como novas ocorrências para o estado do Pará.

Palavras-Chave: Bryophyta; musgos; Ilha de Marajó; novas ocorrências.

The Mosses of the Marajó Island - II - Municipallity of Anajás Pará, Brazil

SUMMARY - Mosses were collected in the municipality of Anajas, on the Marajo Island, in the northen Brazilian state of Pará. This work continues the survey of the Bryophyta flora of this island. Thirty-four species, belonging to 25 genera and 17 families, were identified from the municipality. Calymperaceae and Sematophyllaceae stand out as the families with the greatest number of species (six and five, respectively). The mosses Syrrhopodon leprieurii Mont., Fissidens elegans Brid., Isopterygium subbrevisetum (Hampe) Broth. and Meiothecium boryanum (C. Müll.) Mitt. represent new records for the state of Pará.

Key-Words: Bryophyta; mosses; Marajó Island; new records.

\section{INTRODUÇÃO}

A Illha de Marajó, o maior complexo de ilhas do mundo, com área de 49.606 $\mathrm{km}^{2}$, está dividida em 12 municipios: Afuá, Anajás, Breves, Curralinho, Muaná, São Sebastião da Boa Vista, Chaves, Cachoeira do Arari, Santa Cruz do Arari, Soure, Salvaterra e Ponta de Pedras. Devido a sua localização na foz do rio Amazonas com o Oceano Atlântico, recebe a influência de grandes quantidades de água doce e de marés de água salgada. Possui clima equatorial úmido, com uma estação seca no verão (julho a dezembro) e uma estação chuvosa no inverno (janeiro a junho), relevo plano, com pequenas formações mais elevadas e terrenos inundáveis, tipo depressões e planícies de inundação (várzeas) e diferentes tipos de solos. Todos esses fatores reunidos levam a uma grande variedade de ecossistemas, com flora e fauna especificas. A partir da hipótese de que a brioflora deve refletir essa diversidade, está sendo realizado o estudo dos musgos (Bryophyta) da Ilha de Marajó. Lisboa et al. (1993) abordaram a brioflora da Reserva Ecológica do Bacurizal, em Salvaterra. Lisboa \& Maciel (1994), trataram das briófitas do municipio de Afuá, onde das 31 espécies e uma variedade de musgos identificadas, $28 \%$ foram coletadas pela primeira vez no estado do Pará, o que ressalta a importância dessa pesquisa.

Em continuidade, este trabalho trata dos musgos que ocorrem no municipio de Anajás.

\footnotetext{
I Museu Paraense Emilio Goeldi, Departamento de Botânico, Caixa postal 399, CEP 66.017970, Belém, PA.

2 Estagiária no DBO/MPEG.
} 


\section{ÁREA DE ESTUDO}

Municipio de Anajás - Localiza-se no centro da Ilha de Marajó, fazendo limites com os municípios de Breves, Afuá, Chaves, Ponta de Pedras, Muaná e São Sebastião da Boa Vista. (Fig. 1). É banhada pelo rio Anajás e possui uma área de $7.022,20 \mathrm{~km}^{2}$. Suas coordenadas geográficas são $00^{\circ} 59^{\prime} 21^{\prime \prime} \mathrm{S}$ e $49^{\circ} 56^{\prime} 24^{\prime \prime}$ $\mathrm{W}$, altitude de $10 \mathrm{~m}$ e clima tipo A, superúmido ou tropical chuvoso (Cruz, 1987; Roque, 1994).

Os principais acidentes geográficos são: os rios Anajás, Acarapereira, Aramã e Cururu, os Furos Acarapereira e Camaiani e vários igarapés como Diamante, Fundo e Mocoões. Este último une-se com o rio Anajás, em frente à cidade do mesmo nome (Roque, 1994). Dista $172 \mathrm{~km}$ em linha reta de Belém, com uma altitude de $10 \mathrm{~m}$ na sede do município.

\section{MATERIAL E MÉTODOS}

A coleta do material botânico foi realizada nos meses de fevereiro e março de 1994, num total de 77 amostras de musgos.

As amostras foram coletadas e preparadas segundo metodologia descrita em Yano (1984).

Para a identificação das espécies, foram consultados os trabalhos de Bartram (1949), Florschütz (1964), Griffin III (1979), Edwards (1980), Crum \& Anderson (1981), Florschütz-De Waard (1986), Yano (1992), Allen (1993), Reese (1993), Lisboa (1993) e Sharp et al. (1994). Algumas foram comparadas com material já identificado por especialistas.

O substrato sobre o qual as briófitas foram coletadas segue a classificação de Robbins (1952): corticicolo - troncos e ramos de árvores vivas; epixilo - ramos e troncos caídos e em decomposição; terrestre - superfície do solo ou liter; rupicola-sobre pedras.

O material estudado está depositado no herbário do Museu Paraense Emilio Goeldi (MG).

\section{RESULTADOS}

Foram identificadas 34 espécies de musgos no municipio de Anajás na Ilha de Marajó, distribuídas em 25 gêneros e 17 familias, relacionadas na Tabela 1 , em ordem alfabética de familia.

\section{DISCUSSÃO E CONCLUSÕES}

Dentre as 17 familias identificadas em Anajás, as que apresentaram maior diversidade foram Sematophyllaceae, com quatro gêneros e cinco espécies, Calymperaceae, com dois gêneros e seis espécies e Leucobryaceae com dois gêneros e quatro espécies.

Octoblepharum pulvinatum foi a espécie mais abundante, coletada 33 vezes, seguida por Calymperes erosum (coletada 12 vezes), Calymperes lonchophyllum e Neckeropsis undulata (coletadas 11 vezes). As espécies Syrrhopodon leprieurii, Fissidens elegans, Leucophanes molleri, Isopterygium subbrevisetum 'e Meiothecium boryanum, foram coletadas pela primeira vez para o estado do Pará. Lisboa \& Lima (1997), tratam especificamente de Leucophanes molleri.

Algumas das familias encontradas são comuns a outros municipios da região. Para a Ilha de Marajó, Lisboa \& Maciel (1994) encontraram 15 famílias no municipio de Afuá, das quais 8 são 


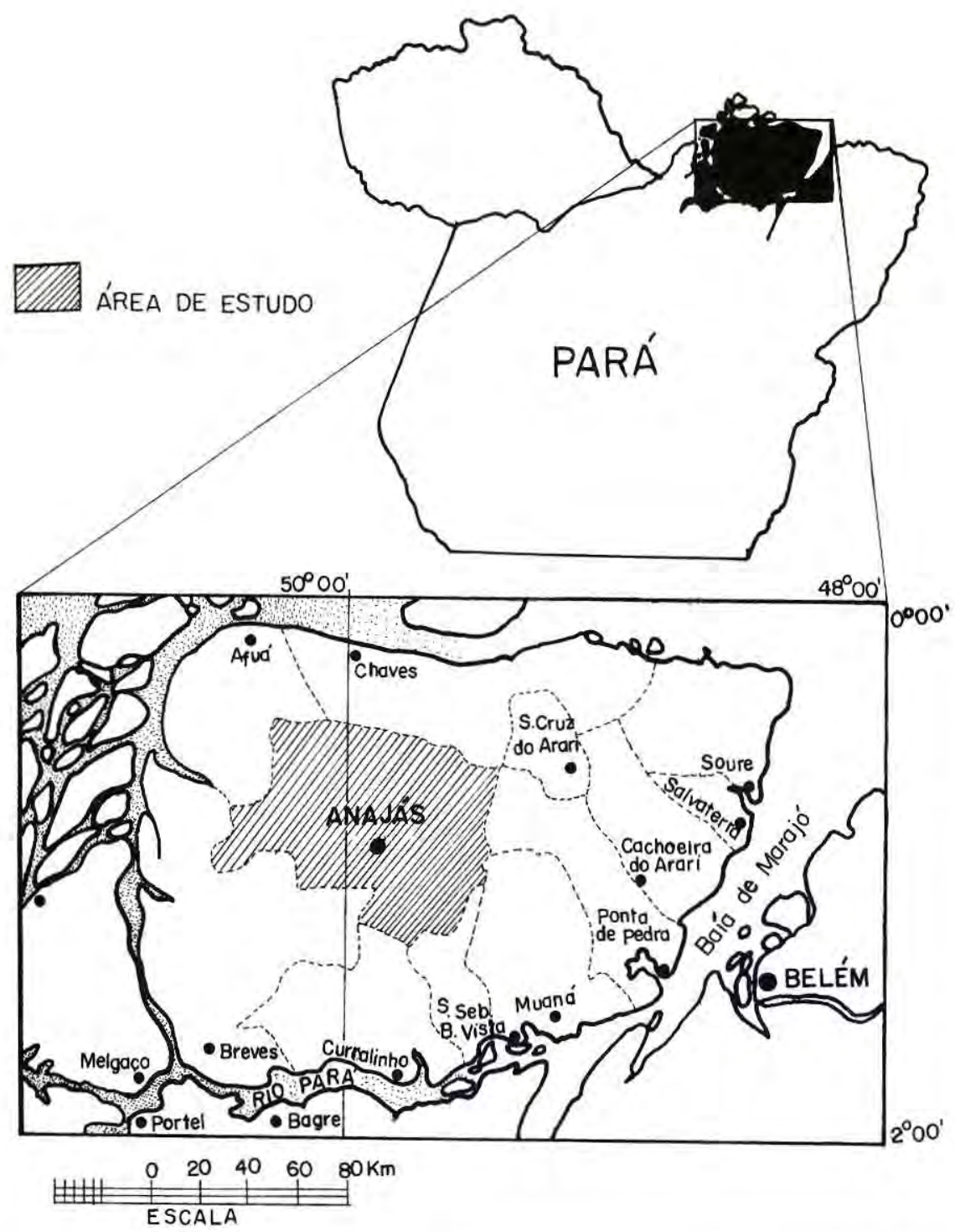

Figura 1. Mapa da Mesorregião do Marajó, destacando a área de estudo, o municipio de Anajás. Observa-se que esse municipio localiza-se centralmente na Ilha de Marajó. 
Tabela 1. Briófitas encontradas em Anajás, llha de Marajó, Pará.

\begin{tabular}{llllllll}
\hline FAMILIA / ESPECCIE & No OCOR. & ECOSSISTEMA & \multicolumn{3}{c}{ SUBSTRATO } \\
\hline & URB & VÁRZ & R & T & C & E \\
\hline
\end{tabular}

\section{BARTRAMIACEAE}

Philonotis uncinata (Schwaegr.) Brid. var.

glaucescens (Hornsch.) Florsch.

BRYACEAE

Bryum apiculatum Schwaegr.

\section{CALYMPERACEAE}

Calymperes afzellii Sw.

C. erosum C. Müll.

C. lonchophyllum Schwaegr.

C. palisotii subsp. richardii (C. Müll.) S. Edwards

Syrrhopodon incompletus Schwaegr.

S. leprieurii Mont.

CALLICOSTACEAE

Callicosta bipinnata (Schwaegr.) C. Müll.

C. evanescens $\mathrm{C}$. Müll.

Callicostella pallida (Hornsch.) Ängstr.

FISSIDENTACEAE

Fissidens elegans Brid.

HOOKERIACEAE

Lepidopilum surinamense C. Müll.

L. stolonaceum C. Müll.

Crossomitrium patrisiae (Brid.) C. Müll.

HYPNACEAE

Vesicularia vesicularis (Schwaegr.) Broth.

var. rutilans (Brid.) Buck

Isopterygium subbrevisetum (Hampe) Broth.

LEUCOBRYACEAE

Leucobryum albidum (Brid. P. Beauv.) Lindb.

L. martianum (Hornsch.) Hampe

Octoblepharum albidum Hedw. var.

violascens $\mathrm{C}$. Müll.

O. pulvinatum (Dozy \& Molk.) Mitt.

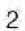

2

5

7

12

11

4

4

1

2

1

5

2

4

1

LEUCODONTACEAE

Henicodium geniculatum (Mitt.) Buck

LEUCOPHANACEAE

Leucophanes molleri C. Müll.

LEUCOMIACEAE

Leucomium strumosum (Hornsch.) Mitt.

METEORIACEAE

Zelometeorium patulum (Hedw.) Manuel

NECKERACEAE

Neckeropsis undulata (Hedw.) Reichdt.

POTTIACEAE

Hyophila involuta (Hook.) Jaeg.

SEMATOPHYLLACEAE

Meiothecium boryanum (C. Müll.) Mitt.

Sematophyllum subsimplex (Hedw.) Mitt.

S. subpinnatum (Brid.) Britt.

Taxithelium planum (Brid.) Mitt.

Trichosteleum sentosum (Sull.) Jaeg.

SPLACHNOBRYACEAE

Splachnobryum obtusum (Brid.) C. Müll.

STEREOPHYLLACEAE

Pilosium chlorophyllum (Hornsch.) C. Müll.

TOTAL

TOTAL

R. rupicola; T. terricola; C. corticicola; E. epíxila; URB. urbano; VARZ. várzea.

6

2

$1 \quad-1$

$33-33$

$1 \quad 1$

7

3

4

11

3

3

11

\section{2}

3

2

43

93

56

2

4

$-$

$-$

1

1

14

2

5

22

1 -

33

$2-$

$2-2 \ldots 11$

$4 \quad 4 \quad-31$

1

312

3

\begin{tabular}{ccccccc}
1 & 1 & - & - & 1 & - & - \\
4 & - & 4 & - & 2 & - & 1 \\
3 & 2 & 1 & $\vdots$ & - & 1 & - \\
1 & - & 1 & - & - & - & 1 \\
1 & - & 1 & & 2 & - & - \\
7 & 7 & - & 5 & 2 & & \\
1 & - & 1 & - & - & 1 & - \\
161 & 22 & 139 & 13 & 10 & 100 & 38 \\
\hline
\end{tabular}

2

22

74

4 
comuns com Anajás (Callicostaceae, Calymperaceae, Hookeriaceae, Leucobryaceae, Meteoriaceae, Neckeraceae, Pottiaceae e Sematophyllaceae). Lisboa et al. (1993), relacionam três familias para Salvaterra, das quais duas (Sematophyllaceae e Calymperaceae), também ocorrem para Anajás. Estas duas familias aparecem também para Afuá, como as familias com maior número de espécies. Calymperes palisotii subsp. richardii, $C$. erosum, Syrrhopodon incompletus var. incompletus e Sematophyllum subsimplex, são espécies comuns aos três municípios.

Na Tabela 1, observa-se claramente que na área urbana a diversidade é bem menor (apenas oito espécies), quando comparada à várzea, onde ocorreram 29 espécies. É interessante destacar que só duas espécies são comuns às duas áreas: Calymperes palisotii subsp. richardii e Sematophyllum subpinnatum. Outras cinco espécies são exclusivas à área urbana: Philonotis uncinata var. glaucescens, Bryum apiculatum, Hyophyla involuta, Meiothecium boryanum e Splachnobryum obtusum.

O substrato preferido é o corticícolo (26 espécies), enquanto o menos requisitado é o rupicolo (4 espécies). As espécies Bryum apiculatum e Splachnobryum obtusum foram coletadas tanto em substrato rupicolo, como terricolo. Calymperes palisotii subsp. richardii ocorreu na terra e sobre árvores vivas.

Os dados encontrados são bem satisfatórios, levando em consideração o número de amostras analisadas. Entretanto não podemos considerar que as espécies encontradas neste trabalho correspondam ao número total de musgos de Anajás.
Novas coletas certamente levarão a um aumento no número de espécies e, possivelmente, até de famílias.

A determinação da diversidade real de musgos de Anajás só estará completa quando, através de sucessivas coletas, em diferentes ecossistemas, não forem mais encontradas novas ocorrências para a região.

\section{Bibliografia citada}

Allen, N.S. 1993. Leucophanaceae. Flora Neotrópica, 59: 1-11

Bartram, E.B. 1949. Mosses of Guatemala. Fieldiana: Botany, Chicago, 25: 1-442.

Crum, H.A.; Anderson, L.E. 1981. Mosses of Eastern North America. New York: Columbia Univ. Press., v.1 e 2.

Cruz, M.E. 1987. Marajo: Essa imensidão de Ilha. São Paulo. 111 p.

Edwards, S.R. 1980. A revision of West tropical African Calymperaceae. 1.Introduction and Calymperes. Journal Bryology, 11 (1): 49-93.

Florschütz, P.A. 1964. The Mosses of Suriname. Part.1. Flora of Suriname, Leiden, 6: 1-127.

Florschütz-De Waard, J. 1964. Musci. Part.II. Flora of Suriname, Leiden, 6: 273-361.

Griffin III, D. 1979. Guia preliminar para as briofitas freqüentes em Manaus e adjacências. Acta Amazonica. Manaus, 9 (3): 1-67. Suplemento.

Lisboa, R.C.L. 1993. Musgos acrocárpicos do Estado de Rondónia. Belém, Museu Paraense Emilio Goeldi, 272p. Coleção Adolpho Ducke.

Lisboa, P.L.B.; Lisboa, R.C.L.; Rosa, N. de A.; Santos, M.R. 1993. Padròes de diversidade Florística na Reserva Ecológica do Bacurizal, em Salvaterra, Ilha de Marajó, Pará. Boletim do Museu Paraense Emilio Goeldi, série Botânica., Belém, 9 (2): 223-248.

Lisboa, R.C.L.; Maciel, U.N. 1994. Musgos da Illha de Marajo-1- Afuá, Pará. Boletim do Museu Paraense Emilio Goeldi, série Botânica., Belém, 1 (10): 43-56. 
Lisboa, R. C. L.; Lima, M. J. de L. 1997. Leucophanaceae, nova família de Bryophyta para o Pará, Brasil. Acta Botanica Brasilica, 11(1): $79-85$.

Reese, D.W. 1993. Calymperaceae. Flora Neotropica, 58: 1-101.

Robbins, R.G. 1952. Bryophyte Ecology of a dune area in New Zealand. Vegetatio, 4: 1-31.

Roque, C. 1994. Microrregião Furo de Breves Anajás. Histórias dos Municípios do Pará. $A$ provincia do Pará, Belém, p.30, 27-28/mar.
Sharp, A.J.; Crum, H.; Eckel, P.M. 1994. The Flora of Mexico. Part two, Memoirs of the New York Botanical Garden, 69: 581-1113.

Yano, O. 1984. Briófitas. In: Fidalgo, O.; Bononi, V.L.R., (Coords.) Técnicas de coleta, preservação e herborização de material botânico. São Paulo, Instituto de Botânica.62p. (manual 4).

Yano, O. 1992. Leucobryaceae (Bryopsida) do Brasil. Tese de Doutorado, Universidade de São Paulo, São Paulo. 318p. 